\title{
EDITORIAL
}

\section{What can BDNF genotype tell us about our memory?}

\author{
Breno S. Diniz \\ Department of Psychiatry and Behavioral Sciences, McGovern Medical School, University of Texas Health Science Center at Houston, \\ Houston, TX, USA.
}

Brain-derived neurotrophic factor (BDNF) is part of the family of neurotrophic factors and is widely expressed in the human brain. BDNF is involved in many neuronal physiological functions, including synaptic plasticity and remodeling, axonal guidance, induction of long-term potentiation, and resilience to neuronal insults, as well as modulation of amyloid- $\beta$ production. ${ }^{1}$ Given its relevance to the maintenance of neuronal functioning, several studies have now investigated its role in the physiopathology of neurodegenerative and neurocognitive disorders.

Single-nucleotide polymorphisms (SNP) in the BDNF gene or in in promoter regions have been associated with changes in protein levels. Of particular interest is the Val66Met (rs6265) SNP. The functional consequence of this substitution is reduced secretion of BDNF, and this SNP has been associated with increased risk of Alzheimer disease..$^{2,3}$

In a study published in this issue, Azeredo et al. found that, among healthy middle-aged and older adults with no evidence of dementia, carriers of the Met allele of the Val66Met polymorphism (homozygous or heterozygous) had significantly worse episodic memory performance. ${ }^{4}$ This provides additional evidence of the relevance of $B D N F$ gene polymorphisms to cognitive performance.

Despite its cross-sectional design, this study elicits provocative thoughts. Could BDNF genotyping be useful for risk prediction of neurocognitive disorders? Can we identify healthy middle-aged subjects that are at higher risk of cognitive decline based on their genetic background and implement preventative interventions to delay the manifestation of dementia? Which are the clinical usefulness and legal limitations of such an approach? While these questions remain unanswered, the results of Azeredo et al. are a first step to moving the field forward towards better models for prognostication of dementia onset in healthy middle-aged and older adults.

\section{Disclosure}

The author reports no conflicts of interest.

\section{References}

1 Teixeira AL, Barbosa IG, Diniz BS, Kummer A. Circulating levels of brain-derived neurotrophic factor: correlation with mood, cognition and motor function. Biomark Med. 2010;4:871-87.

2 Diniz BS, Teixeira AL. Brain-derived neurotrophic factor and Alzheimer's disease: physiopathology and beyond. Neuromolecular Med. 2011; 13:217-22.

3 Forlenza OV, Diniz BS, Teixeira AL, Ojopi EB, Talib LL, Mendonça VA, et al. Effect of brain-derived neurotrophic factor Val66Met polymorphism and serum levels on the progression of mild cognitive impairment. World J Biol Psychiatry. 2010;11:774-80.

4 Azeredo LA, De Nardi T, Levandowski ML, Tractenberg SG, Kommers-Molina J, Wieck A, et al. The brain-derived neurotrophic factor (BDNF) gene Val66Met polymorphism affects memory performance in older adults. Rev Bras Psiquiatr. 2017;39:96-100. 